\title{
Metaphor-based metaheuristics, a call for action: the elephant in the room
}

\author{
Claus Aranha ${ }^{1}$ (D) Christian L. Camacho Villalón ${ }^{2}$ (D) Felipe Campelo ${ }^{3}$. \\ Marco Dorigo $^{2}$ (D) Rubén Ruiz $^{4}$ (D) Marc Sevaux ${ }^{5}$ (D) Kenneth Sörensen ${ }^{6}$ (D) \\ Thomas Stützle ${ }^{2}$ D
}

Received: 29 July 2021 / Accepted: 28 August 2021 / Published online: 30 November 2021

(c) The Author(s) 2021

Taking inspiration from natural behaviors to devise new optimization algorithms has played an important role in the history of the field of metaheuristics (Sörensen et al. 2017). Unfortunately, in the last two decades we have been witnessing a new trend by which dozens of metaphor-based metaheuristics based on the most diverse possible set of natural, artificial, social, and sometimes even supernatural phenomena and behaviors are proposed, without a clear motivation beyond the desire of their authors to publish their papers.

All authors contributed equally to this letter.

Felipe Campelo

f.campelo@aston.ac.uk

Claus Aranha

caranha@cs.tsukuba.ac.jp

Christian L. Camacho Villalón

ccamacho@ulb.ac.be

Marco Dorigo

mdorigo@ulb.ac.be

Rubén Ruiz

rruiz@eio.upv.es

Marc Sevaux

marc.sevaux@univ-ubs.fr

Kenneth Sörensen

kenneth.sorensen@uantwerpen.be

Thomas Stützle

stuetzle@ulb.ac.be

1 University of Tsukuba, Tsukuba, Japan

2 Université Libre de Bruxelles, Bruxelles, Belgium

3 Aston University, Birmingham, UK

4 Universitat Politècnica de València, València, Spain

5 Université Bretagne Sud, Lorient, France

6 University of Antwerp, Antwerp, Belgium 
Despite several attempts (Sörensen 2015; Sörensen et al. 2019; Weyland 2010; Piotrowski et al. 2014; Fong et al. 2016; Camacho Villalón et al. 2019; Camacho Villalón et al. 2020; Swan et al. 2015; Lones 2020; Tzanetos and Dounias 2020) to put an end to the flood of increasingly outlandish, "novel" metaphor-centered metaheuristics, there is still a steady inflow of such papers submitted, and often accepted for publication. For many reasons, we believe this is detrimental to the field of metaheuristics. This letter describes some of the negative effects of publishing such papers in the literature and proposes a necessary action to try to put a limit to this highly undesirable phenomenon.

Useless metaphors The usefulness of the metaphors that inspired the myriad of metaheuristics already published somewhere in the literature is arguably the most questionable aspect of these methods. As demonstrated by the long list of "novel" algorithms gathered by Campelo and Aranha (2021), inventing a metaheuristic that loosely mimics a real-world process is a trivial exercise that does not in itself justify inclusion in the scientific body of literature. Also, it is often the case that the mathematical models derived from the metaphors are modified or omitted in the implementation of the metaheuristics because they result in poorly performing implementations (Melvin et al. 2012; Piotrowski et al. 2014). Indeed, it is not only the fact that using these new metaphors lacks any sound, scientific motivation; but also that, in most cases, the metaphors themselves are not well represented by the resulting computational algorithm (i.e., the metaphor is invariably oversimplified or modified to look like an optimization process even when it is not). Very alarming is the fact that there are numerous examples of metaphor-based metaheuristics in which the metaphor, the mathematical model derived from the metaphor and the implementation of the algorithm are three (almost completely) different things (Camacho Villalón et al. 2020; Camacho Villalón et al. 2021).

Lack of novelty One argument often used to justify introducing a new metaphor-based metaheuristic is that there are novel concepts in the behavior inspiring the algorithm that can be used to solve optimization problems. Unfortunately, there is plenty of evidence that this is rarely the case. In fact, it has become increasingly common to discover, sometimes a few years after publication, that the exact same concepts proposed in the "novel" metaheuristic were proposed before in previously published works (Weyland 2010; Simon et al. 2011; Piotrowski et al. 2014; Camacho Villalón et al. 2019; Camacho Villalón et al. 2020; Tzanetos and Dounias 2020; Camacho Villalón et al. 2021). However, what is invariably new is the non-standard terminology used to present the "novel" metaheuristics. Publishing papers that do not propose anything more than a new terminology to refer to already well-known concepts has pernicious effects, such as (i) creating confusion in the literature, (ii) hindering our understanding of the existing metaphor-based metaheuristics, and (iii) making extremely difficult to compare metaheuristics both theoretically and experimentally.

Poor experimental validation and comparison Biased computational experimentation, the so-called apples to oranges comparison, often comparing "novel" metaphorbased metaheuristics run on recent computers against methods run on older computers, and/or limited experimentation often show a false picture of the performance of a "novel" method (Sörensen 2015; Weyland 2010). In addition to this, rather than testing the "novel" metaheuristic against the best performing algorithms for the considered problem, the comparison is often made with other versions of the very same metaheuristic that is being evaluated or with old algorithms whose performance is far from the state-of-the-art (GarcíaMartínez et al. 2017).

It is important to stress that, even in those cases in which a metaphor-based metaheuristic can be shown to have a very good performance on some optimization problems, 
expressing an optimization method in the non-standard, metaphor-based vocabulary only serves to obfuscate its functioning, making it close to impossible to position the "novel" method in the literature and to discern how it relates to existing methods.

Metaphor-based optimization in application-oriented journals Because of the measures that the field of optimization has been increasingly taking against papers whose only contribution to the literature is a new metaphor upon which an otherwise trivial optimization algorithm is based (see below), authors of such papers are resorting to different outlets, such as journals that publish papers on the topic of the metaphor. For instance, if the metaphor is the mating behaviour of bats, authors will attempt to publish it in specialised journals on bats, or on animal mating behaviour; metaheuristics inspired on improvising musicians will go for journals on music. If the metaphor is the spread of a virus, they will turn to journals on biomedical informatics. The additional advantage of submitting a manuscript to what can only be considered an off-topic journal is that editors and reviewers might not be sufficiently knowledgeable in the specific field of optimization to evaluate the true merits of the paper. Fortunately, this strategy usually does not work and the editors and reviewers quickly realize that the paper is out of scope for their journal. In some cases, regrettably, a paper manages to slip through the net of peer review and gets published. This may happen, e.g., when reviewers are selected from the authors' bubble (based on the list of references or, in some cases, on recommendations of the authors themselves); or when reviewers become blindsided by the metaphor and fail to recognize that the manuscript lacks any meaningful contribution to the state-of-the-art.

The appearance of papers proposing algorithms inspired by often ludicrous processes that do not-by any stretch of the imagination-optimize anything indicates poor scientific housekeeping and, consequently, radiates badly on the research community on metaheuristics. We strongly feel that it is in the best interest of this community that scientific standards in the field are improved, and that papers whose only claim to scientific contribution is having found a new source of inspiration are no longer published. For this reason, we call upon all editors-in-chief in the field to adapt their editorial policies-like several journals such as the Journal of Heuristics (Journal of Heuristics 2015), Swarm Intelligence (Dorigo 2016), and the ACM Transactions on Evolutionary Learning and Optimization (ACM 2021) have already done-and add a statement to the following effect to their submission guidelines:

This journal will not publish papers that propose "novel" metaphor-based metaheuristics, unless the authors (i) present their method using the normal, standard optimization terminology; (ii) show that the new method brings useful and novel concepts to the field; (iii) motivate the use of the metaphor on a sound, scientific basis; and (iv) present a fair comparison with other state-of-the-art methods using state-of-the-art practices for benchmarking algorithms.

Signed by (in alphabetical order; first authors of this letter are indicated by an *):

Claus Aranha*, University of Tsukuba, Japan

Claudia Archetti, ESSEC Business School, France

Anne Auger, Institut National de Recherche en Informatique et en Automatique, France Thomas Bäck, Universiteit Leiden, The Netherlands

Prasanna Balaprakash, Argonne National Laboratory, United States

Thomas Bartz-Beielstein, Technische Hochschule Köln, Germany

Lucas Batista, Universidade Federal de Minas Gerais, Brazil

Kris Braekers, Universiteit Hasselt, Belgium

Juergen Branke, University of Warwick, United Kingdom 
Alexander E. I. Brownlee, University of Stirling, United Kingdom

Christian L. Camacho Villalón*, Université Libre de Bruxelles, Belgium

Felipe Campelo*, Aston University, United Kingdom

Eduardo Carrano, Universidade Federal de Minas Gerais, Brazil

Daniele Catanzaro, Université Catholique de Louvain, Belgium

Marco Chiarandini, Syddansk Universitet, Denmark

Francisco Chicano, Universidad de Málaga, Spain

Carlos A. Coello Coello, CINVESTAV-IPN, Mexico

Ángel Corberán, Universitat de València, Spain

Oscar Cordón, Universidad de Granada, Spain

Yves Crama, Université de Liège, Belgium

Patrick De Causmaecker, Katholieke Universiteit Leuven, Belgium

Javier Del Ser, Universidad del País Vasco, Spain

Gianni Di Caro, Carnegie Mellon University in Qatar, Qatar

Benjamin Doerr, École Polytechnique, France

Carola Doerr, Sorbonne Université, France

Marco Dorigo*, Université Libre de Bruxelles, Belgium

Yu Du, University of Colorado Denver, United States

Anikó Ekárt, Aston University, United Kingdom

Andries Engelbrecht, Stellenbosch University, South Africa

Carlos M. Fonseca, Universidade de Coimbra, Portugal

Alberto Franzin, Université Libre de Bruxelles, Belgium

Margaretha Gansterer, Universität Klagenfurt, Austria

Pablo García Sánchez, Universidad de Granada, Spain

Fred Glover, Meta-Analytics Inc., United States

Maria Gomes, NOVA School of Science and Technology, Portugal

Nikolaus Hansen, INRIA, France

Jin-Kao Hao, Université d'Angers, France

Richard F. Hartl, Universität Wien, Austria

Alfredo G. Hernández-Díaz, Universidad Pablo de Olavide, Spain

Francisco Herrera, Universidad de Granada, Spain

Alain Hertz, Polytechnique Montréal, Canada

Holger Hoos, Universiteit Leiden, The Netherlands

Colin G. Johnson, University of Nottingham, United Kingdom

Ahmed Kheiri, Lancaster University, United Kingdom

Gary A. Kochenberger, Meta-Analytics Inc., United States

Manuel Laguna, University of Colorado Boulder, United States

Adam Letchford, Lancaster University, United Kingdom

Mark Lewis, Missouri Western State University, United States

Xiaodong Li, The University of Melbourne, Australia

Andrea Lodi, Polytechnique Montréal, Canada

Michael Lones, Heriot-Watt University, United Kingdom

Manuel López-Ibáñez, Universidad de Málaga, Spain

Jose A. Lozano, Universidad del País Vasco, Spain

Vittorio Maniezzo, Università di Bologna, Italy

Silvano Martello, Università di Bologna, Italy

Rafael Martí, Universitat de València, Spain

Pedro Martins, Coimbra Business School, Portugal

John McCall, Robert Gordon University, United Kingdom 
Belen Melian, Universidad de La Laguna, Spain

Zbigniew Michalewicz, The University of Adelaide, Australia

Gerardo Minella, Universitat Politecnica de València, Spain

Nenad Mladenovic, Khalifa University United, Arab Emirates

José A. Moreno Pérez, Universidad de La Laguna, Spain

Aneta Neumann, The University of Adelaide, Australia

Frank Neumann, The University of Adelaide, Australia

Thiago Noronha, Universidade Federal de Minas Gerais, Brazil

Gabriela Ochoa, University of Stirling, United Kingdom

Ender Özcan, University of Nottingham, United Kingdom

Federico Pagnozzi, Université Libre de Bruxelles, Belgium

Gisele Lobo Pappa, Universidade Federal de Minas Gerais, Brazil

Luís Paquete, Universidade de Coimbra, Portugal

Eduardo G. Pardo, Universidad Rey Juan Carlos, Spain

Michael Patriksson, Chalmers Tekniska Högskola, Sweden

Federico Perea, Universidad de Sevilla, Spain

Leslie Pérez Cáceres, Pontificia Universidad Católica de Valparaíso, Chile

Riccardo Poli, University of Essex, United Kingdom

Helena Ramalhinho, Universitat Pompeu Fabra, Spain

Andrea Roli, Università di Bologna, Italy

Rubén Ruiz*, Universitat Politècnica de València, Spain

Marc Schoenauer, INRIA, France

Marc Sevaux*, Université Bretagne Sud, France

Christine Solnon, Institut National des Sciences Appliquées de Lyon, France

Michael M. Sørensen, Aarhus Universitet, Denmark

Kenneth Sörensen*, Universiteit Antwerpen, Belgium

Thomas Stützle*, Université Libre de Bruxelles, Belgium

Jerry Swan, NNAISENSE S.A., Switzerland

Eva Vallada, Universitat Politècnica de València, Spain

Greet Vanden Berghe, Katholieke Universiteit Leuven, Belgium

Pieter Vansteenwegen, Katholieke Universiteit Leuven, Belgium

Markus Wagner, The University of Adelaide, Australia

Elizabeth Wanner, Centro Federal de Educação Tecnológica de Minas Gerais, Brazil

Darrell Whitley, Colorado State University, United States

Marino Widmer, Université de Fribourg, Switzerland

John Woodward, Queen Mary University of London, United Kingdom

Rui Zhang, University of Colorado Boulder, United States

Open Access This article is licensed under a Creative Commons Attribution 4.0 International License, which permits use, sharing, adaptation, distribution and reproduction in any medium or format, as long as you give appropriate credit to the original author(s) and the source, provide a link to the Creative Commons licence, and indicate if changes were made. The images or other third party material in this article are included in the article's Creative Commons licence, unless indicated otherwise in a credit line to the material. If material is not included in the article's Creative Commons licence and your intended use is not permitted by statutory regulation or exceeds the permitted use, you will need to obtain permission directly from the copyright holder. To view a copy of this licence, visit http://creativecommons.org/licenses/by/4.0/. 


\section{References}

ACM Transactions on Evolutionary Learning and Optimization. Guidelines for Authors. https://dl.acm.org/ journal/telo/author-guidelines (2021). Version visited last on March 26, 2021

Camacho Villalón, C.L., Dorigo, M., \& Stützle, T. (2018). Why the intelligent water drops cannot be considered as a novel algorithm. In: M. Dorigo, M. Birattari, C. Blum, A.L. Christensen, A. Reina, V. Trianni (eds.) Swarm Intelligence, 11th International Conference, ANTS 2018, Lecture Notes in Computer Science, vol. 11172, (pp. 302-314). Springer.

Camacho Villalón, C. L., Dorigo, M., \& Stützle, T. (2019). The intelligent water drops algorithm: why it cannot be considered a novel algorithm. Swarm Intelligence, 13(3-4), 173-192.

Camacho Villalón, C. L., Stützle, T., \& Dorigo, M. (2020). Grey wolf, firefly and bat algorithms: Three widespread algorithms that do not contain any novelty. In: International Conference on Swarm Intelligence, (pp. 121-133). Springer (2020)

Camacho Villalón, C. L., Stützle, T., \& Dorigo, M (2021). Cuckoo search $\equiv(\mu+\lambda)-$ evolution strategy $-\mathrm{A}$ rigorous analysis of an algorithm that has been misleading the research community for more than 10 years and nobody seems to have noticed. Technical Report TR/IRIDIA/2021-006, IRIDIA, Université Libre de Bruxelles, Belgium.

Campelo, F., \& Aranha, C. (2021). Evolutionary computation bestiary. https:/github.com/fcampelo/ECBestiary (2021). Version visited last on 26 March.

Dorigo, M. (2016). Swarm intelligence: A few things you need to know if you want to publish in this journal. https://www.springer.com/cda/content/document/cda_downloaddocument/Additional_submi ssion_instructions.pdf (2016). Uploaded in November 2016

Fong, S., Wang, X., Xu, Q., Wong, R., Fiaidhi, J., \& Mohammed, S. (2016). Recent advances in metaheuristic algorithms: Does the makara dragon exist? The Journal of Supercomputing, 72(10), 3764-3786.

García-Martínez, C., Gutiérrez, P. D., Molina, D., Lozano, M., \& Herrera, F. (2017). Since CEC 2005 competition on real-parameter optimisation: a decade of research, progress and comparative analysis weakness. Soft Computing, 21(19), 5573-5583.

Journal of Heuristics. Policies on Heuristic Search Research. https://www.springer.com/journal/10732/updat es/17199246 (2015). Version visited last on March 26, 2021.

Melvin, G., Dodd, T. J., \& Groß, R. (2012). Why GSA: a gravitational search algorithm is not genuinely based on the law of gravity. Natural Computing, 11(4), 719-720.

Lones, M. A. (2020). Mitigating metaphors: A comprehensible guide to recent nature-inspired algorithms. SN Computer Science, 1(1), 1-12.

Piotrowski, A. P., Napiorkowski, J. J., \& Rowinski, P. M. (2014). How novel is the novel black hole optimization approach? Information Sciences, 267, 191-200.

Simon, D., Rarick, R., Ergezer, M., \& Du, D. (2011). Analytical and numerical comparisons of biogeography-based optimization and genetic algorithms. Information Sciences, 181(7), 1224-1248.

Sörensen, K. (2015). Metaheuristics-the metaphor exposed. International Transactions in Operational Research, 22(1), 3-18.

Sörensen, K. Sevaux, M., \& Glover, F. (2017). A history of metaheuristics. arXiv preprint arXiv:1704. 00853.

Sörensen, K., Arnold, F., \& Palhazi Cuervo, D. (2019). A critical analysis of the improved Clarke and Wright savings algorithm. International Transactions in Operational Research, 26(1), 54-63.

Swan, J., Adriaensen, S., Bishr, M., \& Burke, et al. (2015). A research agenda for metaheuristic standardization. In: Proceedings of the XI Metaheuristics International Conference, pp. 1-3.

Tzanetos, A., \& Dounias, G. (2020). Nature inspired optimization algorithms or simply variations of metaheuristics? Artificial Intelligence Review,1-22,

Weyland, D. (2010). A rigorous analysis of the harmony search algorithm: How the research community can be misled by a novel methodology. International Journal of Applied Metaheuristic Computing, 12(2), $50-60$.

Weyland, D. (2015). A critical analysis of the harmony search algorithm: How not to solve Sudoku. Operations Research Perspectives, 2, 97-105.

Publisher's Note Springer Nature remains neutral with regard to jurisdictional claims in published maps and institutional affiliations. 